\title{
Globus-M2 spherical tokamak and its mission in developing of compact fusion neutron source
}

\author{
V.B. Minaev ${ }^{1}$, V.K. Gusev ${ }^{1}$, N.V. Sakharov ${ }^{1}$, Yu.V. Petrov ${ }^{1}$, V.I. Varfolomeev ${ }^{1}$, F.V. Chernyshev ${ }^{1}$, \\ N.N. Bakharev ${ }^{1}$, V.V. Dyachenko ${ }^{1}$, N.A. Khromov', G.S. Kurskiev' ${ }^{1}$, A.B. Mineev ${ }^{2}$, V.A. Rozhansky ${ }^{3}$, \\ A.N. Saveliev ${ }^{1}$, P.B. Shchegolev ${ }^{1}$, I.V. Shikhovtsev ${ }^{4}$ \\ ${ }^{1}$ Ioffe Institute, St. Petersburg, Russia, Vladimir.Minaev@mail.ioffe.ru \\ 2 JSC «NIIEFA», St. Petersburg, Russia \\ ${ }^{3}$ Peter the Great St. Petersburg Polytechnic University, St. Petersburg, Russia \\ ${ }^{4}$ BINP SB RAS, Novosibirsk, Russia
}

Development of innovative concepts such as a tokamak based compact fusion neutron source with a nuclear blanket in a beam driven hybrid device needs research and consideration of some principal issues. The overview of the Globus-M spherical tokamak (ST) activities is presented in the report emphasizing the reasons for design and construction of novel high plasma performance machine. The Globus-M2 scientific program and the outcome of predicting simulations for the Globus-M2 plasmas are discussed.

Globus-M [1] is a low aspect ratio compact tokamak $(R=0.36 \mathrm{~m}, a=0.23 \mathrm{~m})$ with high specific ohmic and auxiliary heating power. First plasma was achieved in Globus-M in 1999. The machine has demonstrated practically all of the project objectives ever since. Target design parameters (aspect ratio - 1.5, X-point configuration, vertical elongation -2.2 , traiangularity -0.45 , average density $-1.0 \cdot 10^{20} \mathrm{~m}^{-3}$, plasma current $-0.3 \mathrm{MA}$, auxiliary heating power $-1 \mathrm{MW}$ ) were achieved and some of them overcame [2, 3]. Also Globus-M has demonstrated possibility of reaching very low safety factor at the edge, which was close to 2 . Only the attempt of $0.6 \mathrm{~T}$ magnetic field achieving in 2002 resulted in breakdown and arcing in the upper contact joint of the toroidal field coil. After repairing the toroidal field strength was limited by $0.4 \mathrm{~T}$. Subsequent experiments showed that one of the main factor limiting further enhancements of plasma parameters is a relatively low toroidal magnetic field [4-6].

Globus-M2 [7] is an upgraded version of Globus-M with maximal increase of technical parameters to achieve the promising physical parameter area with sub-fusion temperature value and collisionality much less than unity. All parameters will be reached in compact geometry analogous to the Globus-M magnetic configuration and with the plasma current $0.5 \mathrm{MA}$ and the toroidal magnetic field $1 \mathrm{~T}$.

Magnetic field and plasma current increasing in Globus-M2 led us to complete redesign of the electromagnetic system due to new plasma equilibrium requirements and significant rise of mechanical and thermal loading with respect to Globus-M. High grade materials were used in electromagnetic system manufacturing. Inner segments of the toroidal magnetic field coils and central solenoid were made from cold extruded copper alloy hollow conductors. All poloidal magnetic field coils are water cooled too. The electromagnetic system support structure is enhanced by the upper supporting ring jointed to the lower one by four load-bearing crosspieces which reduces displacement of the toroidal field coil below
$3 \mathrm{~mm}$ under maximal load. Also novel design of coils allows toroidal field ripple minimization down to $0.4 \%$ at the plasma outer boundary. In the Globus-M2 tokamak the vacuum vessel and the in-vessel components remain the same that allows reducing of the project costs. The Globus-M2 machine at the final stage of assembling is shown in figure 1. Power supply upgrade was performed to achieve the ultimately high currents in the toroidal magnet and central solenoid too.

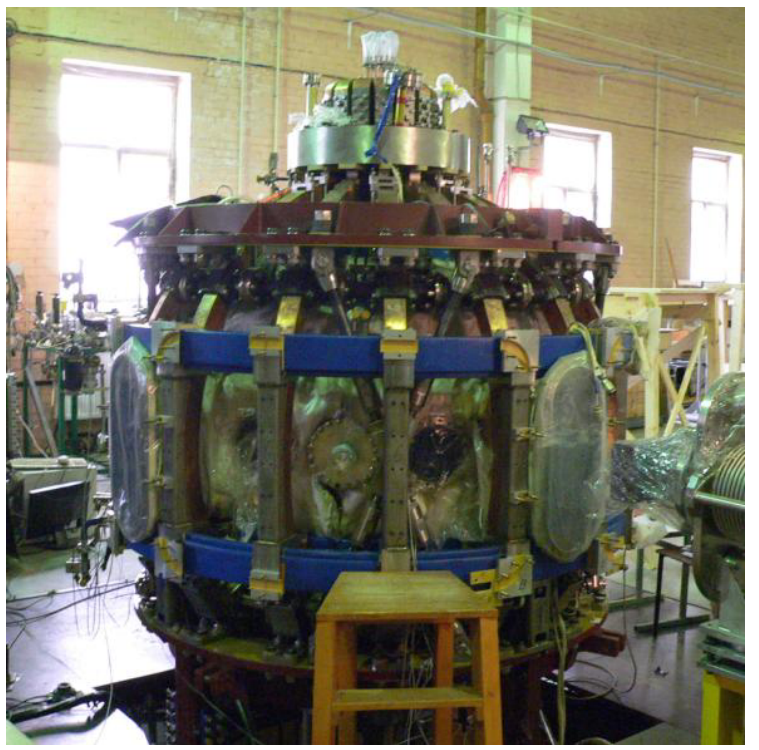

Fig. 1. Globus-M2 assembly

Plasma heating up to the temperatures of several $\mathrm{keV}$ in a compact magnetic configuration specific for the Glopbus-M2 ST requires an intensive auxiliary heating application in conditions of adequate energy confinement. Both the auxiliary heating technique development and the energy confinement improvement are in the focus of Globus-M2 research. A new neutral beam injector with $50 \mathrm{keV}$ hydrogen or deuterium beam of $1 \mathrm{MW}$ power will supplement the "old" one, which will be upgraded up to $40 \mathrm{keV}$ energy, providing additional $1 \mathrm{MW}$ power.

The ion cyclotron plasma heating scenario with light atomic mass minority was successfully tested in Globus-M experiments. New antenna, which is capable for $0.5 \mathrm{MW}$ power launching into Globus-M2 plasma column, has been designed and manufactured. The antenna provides more shaped spectra of exited waves. About $70 \%$ of launched power is located in a range of parallel refractive index 10-40. With increasing of toroidal magnetic field the operating frequency is raised respectively up to $15 \mathrm{MHz}$. 
In turn, that improves antenna-plasma coupling and RF power absorption significantly. Performed numerical modeling predicts essential improving of high energy ion confinement and enhancing of heating efficiency for new experimental conditions.

A problem of non-inductive plasma current start-up and sustainment is principally important for the next generation of "fusion-range" STs, as well as for the tokamakbased compact fusion neutron sources. Neutral beam injection is a well-known method for current drive (CD) complementary to bootstrap current generated by pressure gradients in hot tokamak plasmas. Numerical simulations for Globus-M2 discharges predict the achievement of conditions for better neutral beam CD efficiency and overall plasma performance. The total fraction of noninductive current could reach $40-45 \%$ in full-power NB heated shots.

Low hybrid (LH) electromagnetic waves also demonstrated high efficiency of CD in conventional tokamaks of relatively low cost. Globus-M2 is equipped with a unique system for LHCD and will test a novel scheme for the LH wave launching with poloidal slowing down. The system consists of a klystron generator (0.5 MW, $2.45 \mathrm{GHz}$ ), a 10-channel power splitter and a 10-waveguide grill antenna. Launcher and feeder line construction allows to compare current drive efficiency with toroidal and poloidal slowing down of exited waves for the equal plasma conditions. Also increasing of toroidal magnetic field up to $1 \mathrm{~T}$ has to improve $\mathrm{LH}$ wave propagation significantly.

According to a preliminary modeling the increased magnetic field, plasma current and temperature in Globus-M2 will permit to drive LH current about $200 \mathrm{kA}$ for the poloidal grill orientation and about $460 \mathrm{kA}$ for the toroidal one. Larger driven current in the toroidal case is due to formation of a more extended quasilinear plateau in the electron distribution function compared to the poloidal grill orientation case. It should be noted that the above modeling was done neglecting the inductive electric field, which may play a noticeable role in the LHCD. Taking into account the electric field will be done in the future modeling.

Study of such complex area as scrape of layer and divertor plasma is necessary to provide high duty factor of operation and long-live plasma facing components in a compact fusion device. Comprehensive set of diagnostic tools was prepared for the experiment. Particle flux and electron temperature profiles across the lower divertor plates will be measured by means of an array of $10 \mathrm{em}$ bedded Langmuir probes. A new movable multi-pin Langmuir probe is installed in the equatorial midplane from the low field side. The probe allows obtaining a number of plasma parameters: ion saturation current, floating potential, electron temperature, electron density, radial electric field and Mach number. The IR camera FLIR SC7300M is planned for divertor plate thermal mesurements.

In Globus-M $(0.4 \mathrm{~T}, 200 \mathrm{kA}) \mathrm{D}-\mathrm{D}$ experiments with $26 \mathrm{keV}$ beam the neutron generation rate was about $10^{10}-10^{11} \mathrm{n} / \mathrm{s}$ which corresponded to $10^{10} \mathrm{n} / \mathrm{s} \cdot \mathrm{m}^{2}$ through the vacuum vessel surface. The NUBEAM modeling [8] shows a good agreement with neutron flux measurements on the Globus-M tokamak and predicts a neutron rate increase by two orders of magnitude for the Globus-M2 machine $(1 \mathrm{~T}, 500 \mathrm{kA})$. The main reasons for that enhancement are the electron temperature growth and the substantial improvement of the fast ion confinement which allows application of a new $50 \mathrm{keV} \mathrm{NB}$ injector. The next Globus-3 machine $(R / a=0.6 \mathrm{~m} / 0.36 \mathrm{~m}$; $B_{\mathrm{t}}=1.5 \mathrm{~T}, I_{\mathrm{p}}=1.5 \mathrm{MA}$ ) is currently regarded as a hydrogen prototype of compact tokamak based fusion neutron source. In the $120 \mathrm{keV}, 4 \mathrm{MW}$ D-D NBI discharges modeling predicts a neutron yield level of about $10^{15} \mathrm{n} / \mathrm{s}$ $\left(10^{14} \mathrm{n} / \mathrm{s} \cdot \mathrm{m}^{2}\right)$.

Substantial improvement in plasma parameters is expected for Globus-M2: density over $10^{20} \mathrm{~m}^{-3}$ and temperature in $\mathrm{keV}$ range could be attained with high confidence. High plasma performance together with broad set of auxiliary heating equipment and comprehensive set of diagnostics provide favorable conditions for a compact fusion neutron source research and development.

\section{References}

1. V.K. Gusev, V.E. Golant, E.Z. Gusakov, et al. Globus-M spherical tokamak // Tech. Phys., 44 (1999) 9, 1054.

2. V.K. Gusev, F.V. Chernyshev, V.E. Golant, et al. Density limits and control in the Globus-M spherical tokamak // Nucl. Fusion, 46 (2006) 8, S584-S591.

3. V.K. Gusev, S.E. Aleksandrov, V. Kh. Alimov, et al. Overview of results obtained at the Globus-M spherical tokamak // Nucl. Fusion, 2009, 49 (10), \#104021.

4. V.K. Gusev, R.M. Aminov, A.A. Berezutskiy, et al. Investigation of beam- and wave-plasma interactions in spherical tokamak Globus-M // Nucl. Fusion, 2011, 51 (10), \#103019.

5. V.K. Gusev, E.A. Azizov, A.B. Alekseev, et al. Globus-M results as the basis for a compact spherical tokamak with enhanced parameters Globus-M2 // Nucl. Fusion, 2013, 53 (9), \#093013.

6. V.K. Gusev, N.N. Bakharev, V.A. Belyakov, et al. Review of Globus-M spherical tokamak results // Nucl. Fusion, 2015, 55 (10), \#104016.

7. V.B. Minaev, V.K. Gusev, N.V. Sakharov, et al. Spherical tokamak Globus-M2: design, integration, construction // Nucl. Fusion, 2017, 57 (6), \# 066047.

8. A. Pankin, D. McCune, R. Andre, et al. The tokamak Monte Carlo fast ion module NUBEAM in the National Transport Code Collaboration library // Comput. Phys. Comm., 2004, 159, 157-184. 